\title{
Induced sputum eosinophils and neutrophils and bronchiectasis severity in allergic bronchopulmonary aspergillosis
}

\author{
P.A.B. Wark*, N. Saltos**, J. Simpson*, S. Slater***, M.J. Hensley ${ }^{+}$, P.G. Gibson*
}

\begin{abstract}
Induced sputum eosinophils and neutrophils and bronchiectasis severity in allergic bronchopulmonary aspergillosis. P.A.B. Wark, N. Saltos, J. Simpson, S. Slater, M.J. Hensley, P.G. Gibson. C ERS Journals Ltd 2000.

ABSTRACT: Allergic bronchopulmonary aspergillosis (ABPA) is a hypersensitivity reaction to the fungus Aspergillus fumigatus that may progress to bronchiectasis. The aim of the present study was to characterize airway inflammation in patients with clinically stable ABPA and asthma, and to correlate this with bronchiectasis severity.

Subjects with ABPA and central bronchiectasis (ABPA-CB; $n=16)$ and ABPA with serological evidence alone (ABPA-S; $\mathbf{n}=10$ ) were studied. Comparison groups were $A$. fumigatus-sensitized asthma $(\mathrm{n}=19)$, non- $A$. fumigatus-sensitized asthma $(\mathrm{n}=15)$ and healthy controls $(n=8)$. Hypertonic saline challenge, sputum induction and highresolution computed tomography (HRCT) of the chest were performed.

Sputum eosinophil numbers were markedly elevated in ABPA-CB (median 8.4\%) compared to ABPA-S (2.4\%), A. fumigatus-sensitized asthma (1.8\%), asthma $(1.8 \%)$ and controls $(0.3 \%)(p<0.01)$; sputum eosinophil cationic protein levels were higher in

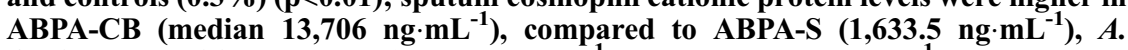
fumigatus-sensitized asthma $\left(1,550.7 \mathrm{ng} \cdot \mathrm{mL}^{-1}\right)$, asthma $\left(309.2 \mathrm{ng} \cdot \mathrm{mL}^{-1}\right)$, and controls (110 $\left.\mathrm{ng} \cdot \mathrm{mL}^{-1}\right)(\mathbf{p}<0.001)$. ABPA-CB also showed increased sputum neutrophil number (median 60.3\%) compared to the other groups (controls $29.3 \%)(\mathrm{p}=0.01)$. The severity of bronchiectasis on HRCT correlated with sputum neutrophil $(\mathrm{r}=0.6)$ and eosinophil number $(r=0.5)$ but not serum immunoglobulin-E levels.

In conclusion, clinically stable allergic bronchopulmonary aspergillosis with bronchiectasis is characterized by an intense heterogenous inflammatory infiltrate consisting of eosinophils and neutrophils, which correlates closely with the severity of bronchiectasis on high-resolution computed tomography. Sputum analysis may be useful in monitoring the course of allergic bronchopulmonary aspergillosis. Eur Respir J 2000; 16: 1095-1101.
\end{abstract}

\begin{abstract}
*Airways Research Centre, **Dept of Respiratory Medicine, John Hunter Hospital, Newcastle ***Hunter Imaging Group, Mayfield, and ${ }^{+}$School of Medical Practice, University of Newcastle, Newcastle, New South Wales, Australia.

Correspondence: P.G. Gibson, Dept of Respiratory Medicine, John Hunter Hospital, Locked Bag 1, Hunter Region Mail Centre, NSW 2310, Australia. Fax: 61249213469
\end{abstract}

Keywords: Allergic bronchopulmonary aspergillosis

Aspergillus fumigatus

asthma

eosinophil

induced sputum

neutrophil

Received: October 201999

Accepted after revision July 102000

This study was supported by the National Health and Medical Research Council of Australia and the Asthma Foundation of New South Wales.
Allergic bronchopulmonary aspergillosis (ABPA) is a serious complication of asthma that is characterized by airway colonization and hypersensitivity to the ubiquitous fungus Aspergillus fumigatus, resulting in severe and chronic asthma, intermittent pulmonary infiltrates, and, in some patients, the development of bronchiectasis and fibrotic lung disease [1]. There is immune activation with raised peripheral blood eosinophil numbers and elevated levels of total immunoglobulin (Ig) E, and A. fumigatus specific IgE and IgG. Ten to twenty-five per cent of asthmatics may be sensitized to A. fumigatus and the full syndrome of ABPA is a complication in up to $2 \%$ of asthmatics [2].

Chronic airway inflammation underlies the disordered airway function found in asthma and bronchiectasis [3, 4]. In asthma, T-helper type 2 lymphocytes (Th2) release interleukin (IL)-5 and orchestrate the eosinophil and mast cell inflammatory infiltrate. In bronchiectasis, chronic infection leads to epithelial damage, release of IL-8 and migration of neutrophils into the airways [4]. Activated neutrophils release myeloperoxidase (MPO), reactive oxygen species [4] and collagenases [5]. This results in chronic airway inflammation and degradation of the connective tissue matrix, leading to bronchiectasis.

ABPA represents a combination of both chronic asthma and bronchiectasis. The role of eosinophils in leading to bronchiectasis in ABPA is unclear. Clarification of this issue may have important implications for the potential effects of eosinophils as a cause of chronic airway damage and remodelling in asthma in general. The present study, therefore, investigated the pattern of airway inflammation in ABPA and related the presence and type of airway inflammation to the severity of bronchiectasis on highresolution computed tomography (HRCT) of the chest. It was hypothesized that ABPA would be characterized by increased sputum total cell counts and a mixed eosinophil/ neutrophil influx, representing the coexistence of asthma and bronchiectasis. Furthermore, it was hypothesized that the intensity of the airway inflammation would be related to the degree of bronchiectasis. In order to control for the separate effects of asthma and aspergillus sensitization (without ABPA), two comparison groups, matched for asthma severity, were included: A. fumigatus-sensitized asthma (without ABPA) and non A. fumigatus-sensitized asthma. 


\section{Methods}

\section{Subjects}

The study group comprised 60 adults with stable asthma and eight healthy controls. The asthmatic subjects were recruited from the respiratory clinic of the John Hunter Hospital and classified using standardized published criteria $[1,2,6,7]$ into the following groups: ABPA and central bronchiectasis (ABPA-CB; $n=16$ ), ABPA with serological evidence alone (ABPA-S; $\mathrm{n}=10$ ), A. fumigatus-sensitized asthma without ABPA $(\mathrm{n}=19)$, and nonA. fumigatus-sensitized asthma $(\mathrm{n}=15)$. Clinic records 1991-1998 were screened for subjects with diagnosed asthma and who showed a positive skin-prick test (SPT) reaction to A. fumigatus. Subjects were excluded if they did not meet objective criteria for asthma, showed no evidence of sensitization to $A$. fumigatus, or reported an exacerbation of asthma within the past 6 weeks. Subjects with cystic fibrosis were also excluded, and, if there was clinical suspicion of this, they went on to undergo sweat electrolyte testing. Eighty-eight subjects were identified during the screening phase. Eleven subjects could not be contacted, four were deceased and nine refused to participate. Four subjects with possible ABPA were assessed and excluded as they did not fulfill the diagnostic criteria: two had mycetoma on HRCT, one probable chronic aspergillus sinus disease and the other postinfective bronchiectasis and asthma but was not sensitized to $A$. fumigatus.

The subjects with asthma who were not sensitized to A. fumigatus, were matched to the subjects with $A$. fumigatus-sensitization and asthma on the basis of age and severity of airway obstruction (forced expiratory volume in one second (FEV1) as a percentage of the predicted value). The eight healthy controls were recruited from hospital staff and were neither sensitized to A. fumigatus nor showed evidence of bronchial hyperresponsiveness (BHR) to hypertonic saline challenge.

\section{Design}

Subjects attended for two visits. At the first visit, a clinical history was elicited followed by assessment of quality of life [8] and venepuncture for immune markers. Spirometry and combined hypertonic saline challenge and sputum induction were then performed, followed by allergy SPT. A. fumigatus-sensitized subjects then attended for HRCT of the thorax, which was performed within 14 days of the clinical assessment. The study was approved by the institutional ethics committee of the Hunter Area Health Service and all subjects gave written informed consent.

\section{Subject classification}

Subjects were evaluated and classified into five groups using the diagnostic criteria of PATTERSON et al. [2] and GrEenBERger et al. [7] (tables 1 and 2): ABPA-CB $(\mathrm{n}=16)$, ABPA-S $(\mathrm{n}=10)$, A. fumigatus -sensitized asthma $(\mathrm{n}=19)$, non A. fumigatus-sensitized asthma $(\mathrm{n}=15)$ and healthy controls $(n=8)$. All cases were reviewed by three respiratory physicians and included only if a consensus was reached. Those with ABPA-CB were required as minimum criteria to show asthma, evidence of $\mathrm{IgE}$ sensitization to A. fumigatus with a positive SPT, a total serum IgE level of $>1,000 \mathrm{IU} \cdot \mathrm{mL}^{-1}$, and evidence of bronchiectasis on HRCT. Those with ABPA-S showed asthma and evidence of $\operatorname{IgE}$ sensitization to A. fumigatus, a total IgE level of $>1,000 \mathrm{IU} \cdot \mathrm{mL}^{-1}$ and the presence of specific IgG to $A$. fumigatus. Those classified as $A$. fumigatus-sensitized asthma and no ABPA showed asthma, and $A$. fumigatus specific IgE but none of the other features of ABPA. Those with asthma alone showed no evidence of sensitization to A. fumigatus. Healthy controls showed neither asthma nor sensitization to $A$. fumigatus. In addition, all subjects underwent tests for precipitating antibodies to A. fumigatus and previous chest radiographs and reports were reviewed for evidence of pulmonary infiltrates.

Asthma was diagnosed using American Thoracic Society criteria and based upon a compatible history together with either BHR (provocative dose of saline causing a $20 \%$ fall in FEV1 of $<15 \mathrm{~mL}$ ) or an improvement in FEV 1 of $>15 \%$ after $200 \mu \mathrm{g}$ salbutamol. Stability of asthma was defined as no deterioration in symptoms or

Table 1. - Subject characteristics

\begin{tabular}{|c|c|c|c|c|c|}
\hline & ABPA-CB & ABPA-S & $A f$-sensitized asthma & Asthma & Control \\
\hline Subjects $n$ & 16 & 10 & 19 & 15 & 8 \\
\hline Males \% & 50 & 40 & 21 & 20 & 12.5 \\
\hline Age yrs & $50.5 \pm 12.6$ & $60.3 \pm 10.7$ & $49.4 \pm 14.8$ & $50.2 \pm 18.6$ & $34.6 \pm 6.4 * * *$ \\
\hline Atopic $\mathrm{n}(\%)^{+}$ & $11(69)$ & $8(80)$ & $14(74)$ & $11(73)$ & $4(50)^{3}$ \\
\hline Smokers \% & 0 & 0 & 21 & 20 & 25 \\
\hline FEV1\% pred & $52.4 \pm 34.7$ & $57.2 \pm 15.8$ & $61.3 \pm 21.9$ & $68.6 \pm 12.2$ & $105.1 \pm 11.6^{* * *}$ \\
\hline SGRQ score $\%^{+}$ & $46.9 \pm 15.6$ & $38.4 \pm 13.3$ & $47.5 \pm 17.2$ & $35 \pm 17.1$ & NA \\
\hline ICS $\mu \mathrm{g}$ BDP·day ${ }^{-1 \#}$ & $2000(1500-3000)$ & $2000(2000-2400)$ & $1600(1000-4000)$ & $1600(1500-1800)$ & NA \\
\hline Oral steroids \% & 31 & 20 & 22 & 10 & NA \\
\hline OCS use in last year \% & 75 & 80 & 58 & 60 & NA \\
\hline
\end{tabular}

Data are presented as absolute values, mean $\pm \mathrm{SD}$ or median (interquartile range). ${ }^{+}$: positive skin-prick test with weal diameter $>2 \mathrm{~mm}$ on exposure to aeroallergens other than Aspergillus species (Alternaria tenuis, Penicillium species, Dermatophagoides farinae, D. pteronyssinus, cat, dog, feather, cockroach and grass mix); ${ }^{*}:$ higher score indicates greater impaction on quality of life; ${ }^{\#}$ : inhaled corticosteroid (ICS) doses were standardized to beclomethasone dipropionate (BDP): $1 \mu \mathrm{g}$ BDP $=1 \mu \mathrm{g}$ budesonide $=0.5 \mu \mathrm{g}$ fluticasone propionate. ABPA-CB: allergic bronchopulmonary aspergillosis (ABPA) and central bronchiectasis; ABPA-S: ABPA with serological evidence alone; Af: Aspergillus fumigatus; FEV1: forced expiratory volume in one second; SGRQ: St Georges Respiratory Questionnaire; OCS: oral corticosteroid; NA: not assessed; \% pred: percentage of the predicted value. ${ }^{* * *}: \mathrm{p}<0.001 ;{ }^{\S}: \chi^{2}=2.76, p=0.6$. 
Table 2. - Serum immunological markers

\begin{tabular}{lccccc}
\hline & ABPA-CB & ABPA-S & Af-sensitized asthma & Asthma & p-value \\
\hline Total IgE IU.mL $\mathrm{mL}^{-1}$ & $1792(403-5000)^{*}$ & $2158(919-3632)^{*}$ & $146(62-452)$ & $156(108-200)$ & $<0.001$ \\
IgE to Af IU.mL & $4(3-5)^{*}$ & $1(0-3)$ & 0 & 0.02 \\
IgG to Af IU.mL I $^{-1}$ & $49.5(56.7-60.9)$ & $39.8(38.3-44.9)$ & $<10$ & $<10$ & NA \\
Positive precipitins $\%$ & 50 & 40 & 0 & 0 & NA \\
Serum eosinophils $10^{6}$ cells $\cdot \mathrm{dL}^{-1}$ & $0.6(0.2-1.2)^{*}$ & $0.5(0.2-0.5)$ & $0.2(0.1-0.4)$ & $0.2(0.1-0.4)$ & 0.04 \\
\hline
\end{tabular}

Data are presented as median (interquartile range). ABPA-CB: allergic bronchopulmonary aspergillosis (ABPA) and central bronchiectasis; ABPA-S: ABPA with serological evidence alone; Af: Aspergillus fumigatus; Ig: immunoglobulin; NA: not assessed. *: $\mathrm{p}<0.05$ versus Af-sensitized asthma and asthma.

peak flow or no increased use of bronchodilator medication in the preceding 6 weeks. Sensitization to A. fumigatus was determined by an immediate reaction to skin-prick testing (weal of $>3 \mathrm{~mm}$ ), carried out using a 1:10 weight/ volume dilution of A. fumigatus (Bayer Australia Ltd., Pymble, Australia).

\section{Measurements}

Quality of life was assessed using the St Georges Respiratory Questionnaire. Clinical details were elicited, including medication history, frequency and severity of asthma symptoms, allergy history, age at diagnosis of asthma and frequency of hospitalization due to asthma.

Full blood and differential (including eosinophil) cell counts were obtained using a Coulter Gen-S (BeckmanCourter, Australia Pty Ltd, Sydney, Australia). Serum IgE was assayed by Unicap (Pharmacia-Upjohn Diagnostics, $\mathrm{AB}$, Uppsala, Sweden), and specific precipitating IgG to $A$. fumigatus in serum was detected using a double gel diffusion assay (Microgen Bioproducts, Camberley, UK). Serum IgE to A. fumigatus were measured using a Pharmacia CAP immunoassay (Kabi Pharmacia Diagnostics, AB, Uppsala, Sweden). Serum IgG to A. fumigatus (IgGAf) was measured by enzyme-linked immunosorbent assay (ELISA) (Genesis Diagnostics, Cambridgeshire, UK).

\section{Pulmonary function tests}

Spirometry (Minato Autospiro AS-600; Minato Medical Science Co Ltd., Osaka, Japan) and bronchial provocation testing with hypertonic $(4.5 \%)$ saline were performed as previously described [9], using a DeVilbiss 2000 ultrasonic nebulizer (Devilbiss, Oregon, PA, USA) and a Hans Rudolph 2700 two-way nonrebreathing valve box (Hans Rudolph Inc., Kansas City, KS, USA) with a rubber mouthpiece and nose clips. The nebulizer output was $1.8 \mathrm{~mL} \cdot \mathrm{min}^{-1}$ and mass median aerodynamic diameter particle size $<5 \mu \mathrm{m}$. Sputum was induced during the hypertonic saline challenge as previously described [9]. Subjects rinsed their mouth before the procedure to help minimize squamous cell contamination, and were asked to cough and expectorate into a container between each dose of nebulized saline.

\section{Sputum analysis}

Lower respiratory sputum portions were selected from saliva and processed as previously described [9]. Briefly, selected sputum was dispersed by adding four volumes of $0.1 \%$ dithiothreitol (DTT; Sputolysin 10\%; Calbiochem Corporation, La Jolla, CA, USA), mixed in a rotating waterbath for $30 \mathrm{~min}$ at $37^{\circ} \mathrm{C}$, and then four volumes of phosphate-buffered saline (PBS) added. The suspension was filtered through $60-\mu \mathrm{m}$ nylon gauze (Millipore; North Ryde, New South Wales, Australia) and the total cell count (TCC) of nonsquamous cells and viability of the filtrate were determined. The filtrate was centrifuged for $10 \mathrm{~min}$ at $200 \times g$ and the supernatant aspirated and stored in Eppendorf tubes at $-70^{\circ} \mathrm{C}$. The cell pellet was resuspended in PBS to give a concentration of $1 \times 10^{6}$ cells $\cdot \mathrm{mL}^{-1}$ and placed into the cups of a Shandon II cytocentrifuge (Shandon Cytospin; Sewickley, PA, USA). A differential count was obtained from 400 cells counted on May-Grünwald Giemsa-stained cytopreps. Eosinophils were enumerated from slides stained with chromotrope $2 \mathrm{R}$ in the same fashion.

The concentration of eosinophil cationic protein (ECP) was determined by radioimmunoassay (RIA; Kabi Pharmacia Diagnostics, AB) and MPO was assayed by ELISA (Oxis International, Portland OR, USA), with standard curves based on dilutions of purified ECP and MPO respectively. The limits of detection of the fluid phase assays were ECP $2 \mu \mathrm{g} \cdot \mathrm{mL}^{-1}$ and MPO $1.6 \mathrm{ng} \cdot \mathrm{mL}^{-1}$.

\section{High-resolution computed tomography assessment of} the chest

All patients who were sensitized to A. fumigatus underwent HRCT of their chest with a Toshiba TCT900S (Toshiba Medical Division; Tokyo, Japan), using a section width of $2 \mathrm{~mm}, 120-140 \mathrm{kV}, 200 \mathrm{~mA}$ and a scan time of 1-2 $\mathrm{s}$ at the end of inspiration. The images were viewed with lung windows at $700 \mathrm{HU}$ and a width of 1,200 HU. These films were reported and scored by a radiologist (S. Slater), according to the following categories [10]: severity of bronchiectasis based on lumenal diameter, degree of peribronchial thickening, extent of bronchopulmonary segments involved by bronchiectasis, number of bronchopulmonary segments involved with mucus plugging, number of bronchopulmonary segments involved with sacculations, generations of bronchial divisions involved with bronchiectasis or mucus plugging, number of bullae, emphysema and areas of collapse or consolidation. Each of these categories was given a score ranging $0-2$ or $0-3$, the higher number representing more severe disease. A cumulative score was obtained from the sum of all the categories, with a maximum score of 25 and a minimum of 0 . 


\section{Statistical analysis}

Statistical analysis was carried out using STATA (State Corporation, College Station, TX, USA). For data that were normally distributed, a two-tailed unpaired t-test and one-way analysis of variance (ANOVA) were used. Differences in proportions between groups were analysed by Chi-squared test. The indices of airway inflammation measured in induced sputum were not normally distributed and when reported are expressed as medians and interquartile ranges. The data were analysed after log transformation by one-way ANOVA. If the distribution was not normal despite transformation then a KruskalWallis test or a Wilcoxon rank sum test was used. Univariate relationships between continuous variables were analysed using Pearson's correlation test or Spearman's rank correlation coefficient. Relationships between inflammatory cells and fluid phase measurements were determined using absolute cell counts. Multivariate analysis was carried out on variables after log transformation. A p-value of $<0.05$ was regarded as statistically significant.

\section{Results}

The subjects with ABPA-CB, ABPA-S, A. fumigatussensitized asthma and asthma had moderate-to-severe asthma. Their FEV1 was reduced to $\sim 60 \%$ pred (table 1 ). These four asthma groups were comparable in degree of airway obstruction, daily dose of inhaled corticosteroid and proportions of either current or past smokers. Similarly, there was no significant difference between the groups in quality of life score (higher score associated with increased severity of disease). Subjects with ABPA$\mathrm{CB}$ and ABPA-S had a similar degree of immune activation with higher levels of total serum $\operatorname{IgE}$, specific $\operatorname{IgE}$ and IgG to $A$. fumigatus compared to A. fumigatussensitized asthma and nonsensitized asthmatics $(\mathrm{p}<0.001)$ (table 2). Peripheral blood eosinophil numbers were higher in those with ABPA-CB ( $p=0.04)$.

\section{Radiological change}

Those with ABPA-CB were distinguishable from the other groups by an overall greater severity of disease on HRCT (table 3). This was due to the presence of bronchiectasis, mucus plugging in the large airways and a greater proportion of subjects with areas of segmental or subsegmental collapse. The changes present on HRCT in those with ABPA-S were similar to those in those with
A. fumigatus-sensitized asthma alone. Those with $A$. fumigatus-sensitized asthma did not have a history of previous chest radiograph infiltrates, whereas this was present to a similar degree in both groups with ABPA.

\section{Induced sputum}

The sputum TCC was significantly higher in those with ABPA-CB compared to each of the other groups $(\mathrm{p}<0.001)$, including those with ABPA-S $(\mathrm{p}=0.02)$ (table 4). Sputum eosinophil numbers were also significantly higher in ABPA-CB ( $p=0.002)$. ECP in sputum supernatant was used as a marker of eosinophil degranulation, and those with ABPA-CB had significantly higher levels of ECP than all of the other groups $(p<0.001)$, except those with ABPA-S. Those with ABPA-S had higher ECP levels than the controls $(p<0.05)$. Levels of ECP correlated closely with the number of sputum eosinophils $(\mathrm{r}=0.9)$. Interestingly, there was also a close correlation between the number of sputum neutrophils and ECP level $(\mathrm{r}=0.7)$. Sputum eosinophil numbers also correlated with serum eosinophil numbers $(\mathrm{r}=0.6)$. There was no correlation between total serum IgE levels and sputum eosinophil numbers or ECP levels.

Sputum neutrophil numbers were significantly higher in ABPA-CB $(\mathrm{p}<0.001)$, and, although those with ABPA-S and $A$. fumigatus-sensitized asthma tended to show higher neutrophil numbers than controls, this did not reach significance. Sputum MPO levels tended to be higher in those with ABPA and A. fumigatus-sensitized asthma, but this did not reach significance $(\mathrm{p}=0.06)$.

Subjects using oral corticosteroids tended to have a higher TCC, sputum eosinophil numbers and sputum ECP level, but this did not reach statistical significance. Smoking status had no impact on the markers of airway inflammation in sputum $(\mathrm{p}>0.05)$.

\section{Relationship of airway inflammation to severity of bronchiectasis}

In the groups sensitized to A. fumigatus, there were significant positive relationships between a higher HRCT score (more severe disease) and increased sputum TCC $(\mathrm{r}=0.6)$, sputum eosinophil numbers $(\mathrm{r}=0.5)$, sputum neutrophil numbers $(\mathrm{r}=0.6)$ (fig. 1) and sputum ECP level $(\mathrm{r}=0.5)$. Sputum MPO level was not related to HRCT score. Increased serum eosinophil numbers and total IgE level did not correlate with HRCT score. The relationship between HRCT score and inflammatory markers

Table 3. - Radiographic features in the groups sensitized to aspergillus

\begin{tabular}{lcccc}
\hline & ABPA-CB & ABPA-S & Af-sensitized asthma & p-value \\
\hline Bronchiectasis \% & 100 & 0 & 0 & $<0.001$ \\
Collapse/consolidation \% & 75 & 20 & 23 & 0.5 \\
Peribronchial thickening \% & 81 & 20 & 23 & $<0.0001$ \\
Mucus plugging \% & 63 & 0 & 0 & $<0.001$ \\
HRCT score & $8.5(7-12)$ & $1.5(1-2)$ & $1(0-3)$ & $<0.0001$ \\
History of previous infiltrates on CXR \% & 71 & 67 & 0 & 0.09 \\
\hline
\end{tabular}

Data are presented as absolute values or median (interquartile range). ABPA-CA: allergic bronchopulmonary aspergillosis (ABPA) and central bronchiectasis; ABPA-S: ABPA with serological evidence alone; Af: Aspergillus fumigatus; HRCT: high-resolution computed tomography; CXR: chest radiography. 
Table 4. - Indices of airway inflammation in induced sputum

\begin{tabular}{lcccccc}
\hline & ABPA-CB & ABPA-S & Af-sensitized asthma & Asthma & Control & p-value \\
\hline TCC $10^{6}$ cells $\cdot \mathrm{mL}^{-1}$ & $13.7(4.4-29.4)^{*,+}$ & $3.5(1.8-4.3)$ & $3.6(0.9-9.3)$ & $2.3(1.2-3.2)$ & $1.4(1.3-1.4)$ & $<0.01$ \\
Eosinophils \% & $8.4(3-14.7)^{*}$ & $2.4(2.3-3.8)^{*}$ & $1.8(0.3-6)$ & $1.8(1.5-2)$ & $0.3(0.25-0.3)$ & $<0.01$ \\
Neutrophils \% & $60.3(43.3-77)^{+}$ & $34.5(27.5-42.4)$ & $50.3(20.3-67)$ & $25.9(20.6-27)$ & $29.3(29.2-29.4)$ & 0.01 \\
Macrophages \% & $24.9(9.3-37.5)$ & $56.1(50.0-73)$ & $38.7(10.3-72)$ & $54.7(51.0-69.7)$ & $66.4(63.8-68.9)$ & 0.1 \\
Epithelial cells \% & $1.0(0-6.4)$ & $1.1(0-6.7)$ & $0.98(0-8.5)$ & $0.8(0.3-3.4)$ & $0.8(0.8-0.9)$ & 0.99 \\
Lymphocytes \% & $0.25(0-2.75)$ & $0(0-0.7)$ & $0(0-0.8)$ & $0.5(0-0.8)$ & $0.25(0.26-0.28)$ & 0.6 \\
ECP ng.mL ${ }^{-1}$ & 13706 & 1634 & 1551 & 309 & $(110$ & $<0.01$ \\
MPO ng.mL ${ }^{-1}$ & $(23345-40514)^{*,+, \#}$ & $(329-2682.7)^{*}$ & $(338.5-8099.6)$ & $(187-1066.2)$ & $(98.9-121.1)$ & 0.06 \\
& 219.2 & 320 & 359 & 187.8 & 65.1 & $(54.5-75.8)$ \\
\hline
\end{tabular}

Data are presented as median (interquartile range). ABPA-CB: allergic bronchopulmonary aspergillosis (ABPA) and central bronchiectasis; ABPA-S: ABPA with serological evidence alone; Af: Aspergillus fumigatus; TCC: total cell count; ECP: eosinophil cationic protein; MPO: myeloperoxidase. ${ }^{*}: \mathrm{p}<0.05$ versus control; ${ }^{+}: \mathrm{p}<0.05$ versus asthma; ${ }^{*}: \mathrm{p}<0.05$ versus $\mathrm{ABPA}-\mathrm{CB}$.

was also examined in the two groups with ABPA-CB and ABPA-S. The associations remained for sputum eosinophil numbers $(\mathrm{r}=0.6, \mathrm{p}=0.002)$ and sputum neutrophil numbers $(\mathrm{r}=0.6, \mathrm{p}<0.001)$ but was weakened for sputum ECP level $(\mathrm{r}=0.4, \mathrm{p}=0.04)$.

A multivariate linear regression model was used to determine which variables were associated with HRCT score in subjects with ABPA. The model with the best fit predicted $58.5 \%$ of the variation in HRCT score in the subjects with ABPA, and included sputum neutrophil number, sputum eosinophil number, serum eosinophil number, total serum IgE level, sex, age, smoking status
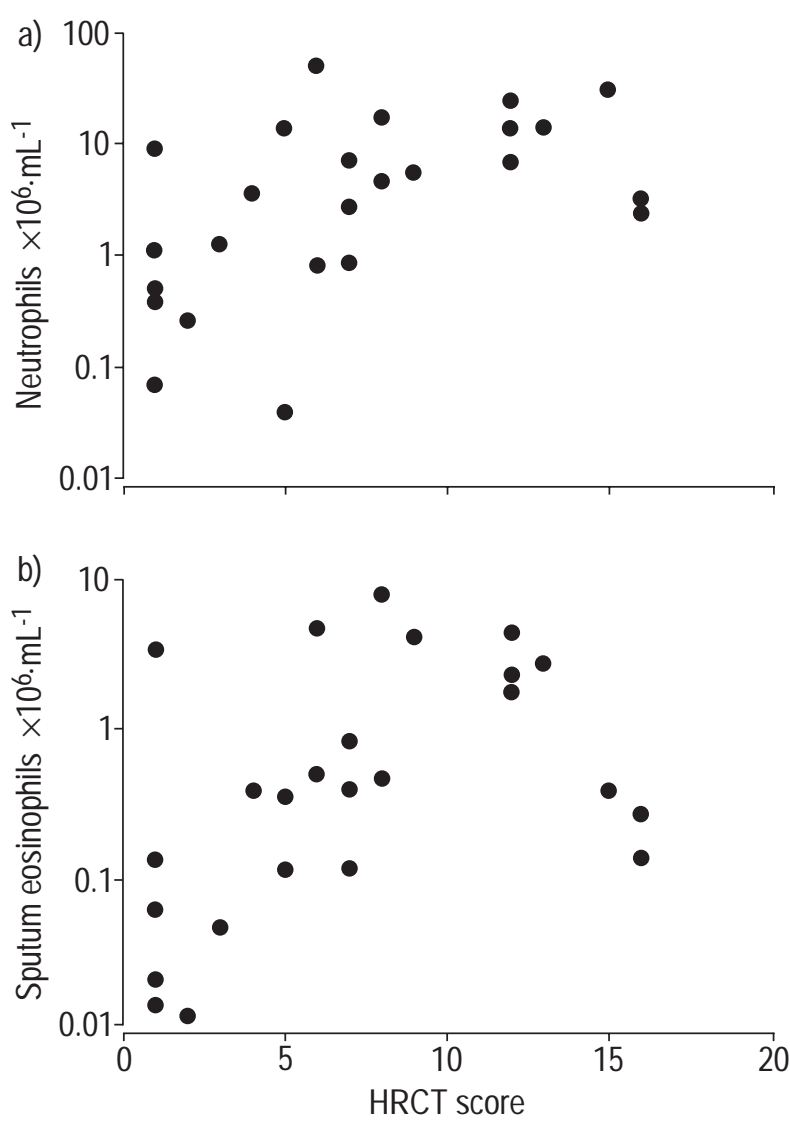

Fig. 1. - Relation between: a) sputum neutrophil number; and b) sputum eosinophil number and high-resolution computed tomography (HRCT) score. and oral corticosteroid use. In this model, increased sputum neutrophil number $(\beta=0.34, \mathrm{p}=0.01)$ was strongly associated with higher HRCT scores and there was a trend towards increased sputum eosinophil number also being associated $(\beta=0.22, p=0.07)$. Higher levels of total serum IgE were associated with lower HRCT scores $(\beta=-0.25$, $\mathrm{p}=0.03)$. The addition of sputum ECP and MPO levels had no significant effects and worsened the fit of the model. This may have been because there were close correlations between these variables and sputum eosinophil and neutrophil numbers.

\section{Discussion}

The present study shows that the airway inflammatory response in stable ABPA with bronchiectasis is characterized by an increased intensity of airway inflammation, with elevated sputum eosinophil as well as neutrophil numbers and evidence of marked eosinophil degranulation with elevated sputum ECP levels. In addition, it has been demonstrated that the degree of airway inflammation present predicts the severity of disease on HRCT, whereas markers of systemic immune activation do not.

The diagnosis of ABPA can be problematic. GREENBERGER and coworkers $[2,7,11]$ have continued to refine the diagnostic criteria with time. The present study applied their classification system since it is standardized and widely accepted. To minimize selection bias, subjects were recruited on the basis of $A$. fumigatus sensitization, and not their previous diagnosis of ABPA. Furthermore, subjects were included only after agreement between three respiratory physicians. None of the subjects with ABPA-CB had a history of recurrent pulmonary infections in childhood to suggest the development of bronchiectasis prior to their diagnosis of asthma. Although all but one of the subjects had developed asthma aged $<20 \mathrm{yrs}$ and had histories consistent with asthma prior to their development of ABPA, it cannot be determined whether those with ABPA developed bronchiectasis as a consequence of $A$. fumigatus or airway inflammation secondary to asthma alone from the results of the present study. However, it can be demonstrated that the airway inflammation in ABPA-CB is distinct from that in ABPA$\mathrm{S}$ and A. fumigatus-sensitized asthma. In order to test the hypothesis that bronchiectasis in asthma is a consequence of A. fumigatus colonization, and that ABPA-S is a 
precursor condition and airway inflammation alone is not responsible, would require a prospective cohort study.

ABPA was originally described with central bronchiectasis as a key feature. The syndrome has recently been expanded to include subjects with intense immune activation but no bronchiectasis, termed ABPA-S [7]. The present study showing that those with ABPA-CB and ABPA-S were similar in the intensity of their systemic immune response to $A$. fumigatus and had a similar frequency of radiographic infiltrates is consistent with other descriptions [7]. However, the pattern and intensity of airway inflammation in ABPA-S was different to that in ABPA-CB. KAUFFMAN et al. [12] has proposed that simple allergy to aspergillus leading to asthma occurs with a typical Th2 response and consequent eosinophilia, whereas ABPA represents a more complex reaction with superimposed colonization of epithelial surfaces by $A$. fumigatus and consequent epithelial activation by aspergillus proteases and release of potent neutrophil attractants such as IL-8. Prior examinations of bronchoalveolar lavage fluid have identified elevated eosinophil numbers but no increase in neutrophil number in ABPA [13-16]. However, pathological sections from excised lungs of subjects with ABPA show a mixed neutrophil/eosinophil infiltrate, associated with bronchocentric granulomatosis and bronchiectasis [17]. The present data are consistent with these hypotheses, and clearly demonstrate that inflammation in ABPA-CB involves a mixed neutrophil/ eosinophil infiltrate and that it differs from A. fumigatussensitized asthma. Uncomplicated stable asthma is not associated with an increase in sputum TCC, and nor is it associated with elevated sputum neutrophil numbers [18], but these are features of bronchiectasis [19]. There was also evidence of intense eosinophil activation in ABPACB despite stable clinical disease, with a median sputum ECP level of $13,706 \mathrm{ng} \cdot \mathrm{mL}^{-1}$. This was 120 times higher than in the controls and 40 times higher than in those with stable asthma. Median levels of $4,609 \mathrm{ng} \cdot \mathrm{mL}^{-1}$ have been reported during acute exacerbations of asthma [20], which is still considerably less than in the present stable ABPA-CB subjects.

The present results suggest an additive effect of neutrophils on eosinophilic airway inflammation, since there was a close correlation between sputum neutrophil and eosinophil numbers $(r=0.7)$, and between the number of neutrophils and the degree of eosinophil activation (ECP level and neutrophil numbers, $\mathrm{r}=0.7)$. When neutrophils and eosinophils were included in the regression analysis as separate parameters, each was significant, and, when added together to produce a single variable reflecting inflammatory cell numbers, this combined variable explained more of the variability in HRCT scores, $(\beta=0.257,95 \%$ confidence interval $0.121-0.393$, adjusted $\mathrm{R}^{2}=0.612$ ).

The extent of lung damage was assessed using a validated HRCT score [10]. Prior work relating peripheral blood immunological markers to radiographic features in ABPA has given variable results. In one study, aspergillus precipitin but not $\operatorname{IgE}$ levels were related to radiographic features [21], and, recently, KIELY et al. [22] reported that peripheral blood eosinophil number and A. fumigatus antibody levels were related to chest radiography score. The present study extends these observations by directly showing a quantitative relationship between sputum neutrophil and eosinophil numbers and the extent of disease present on HRCT of the chest. Systemic markers of disease in ABPA (total IgE level, serum eosinophil numbers and serum $A$. fumigatus antibody levels) did not predict the severity of disease on HRCT. This has important implications as it is currently recommended that disease activity be monitored by measuring serum eosinophil number and total IgE level [23]. It may be that induced sputum would be of use in monitoring ABPA.

The present study has a number of important implications. Airway inflammation in stable allergic bronchopulmonary aspergillosis with bronchiectasis is more intense and heterogenous than in stable asthma. A distinction can be made between those with allergic bronchopulmonary aspergillosis and central bronchiectasis and those with allergic bronchopulmonary aspergillosis with serological evidence alone. The present study emphasizes that airway inflammation may be important in the genesis of progressive bronchiectasis in allergic bronchopulmonary aspergillosis, by showing intense eosinophilic activation even in stable clinical disease and indicating the possibility of neutrophil-mediated damage. In addition the severity of disease on computed tomography correlates closely with airway inflammation markers measured in sputum. It is more closely correlated than with systemic markers of immune activation such as serum immunoglobulin E levels or serum eosinophil numbers in stable disease; this implies that direct measures of sputum inflammation may be a more sensitive predictor of disease activity.

\section{References}

1. Varkey B. Allergic bronchopulmonary aspergillosis: clinical perspectives. Immunol Allergy Clin N Am 1998; 18: 479-501.

2. Patterson R, Greenberger G, Halwig M, Liotta J, Roberts M. Allergic bronchopulmonary aspergillosis. Arch Intern Med 1986; 146: 916-918.

3. Nadel JA, Busse WW. Asthma. Am J Respir Crit Care Med 1998; 157: S130-S138.

4. Wilson R, Dowling RB, Jackson AD. The biology of bacterial colonization and invasion of the respiratory mucosa. Eur Respir J 1996; 9: 1523-1530.

5. Sepper R, Konttinen YT, Ding Y, Takagi M, Sorsa T. Human neutrophil collagenase (MMP-8), identified in bronchiectasis BAL fluid, correlates with severity of disease. Chest 1995; 107: 1641-1647.

6. Bateman ED. A new look at the natural history of aspergillus hypersensitivity in asthmatics. Respir Med 1994; 88: 323-325.

7. Greenberger P, Miller T, Roberts M, Smith L. Allergic bronchopulmonary aspergillosis in patients with and without evidence of bronchiectasis. Ann Allergy 1993; 70 : 333-338.

8. Wilson CB, Jones PW, O'Leary CJ, Cole PJ, Wilson R. Validation of the St George's Respiratory Questionnaire in bronchiectasis. Am J Respir Crit Care Med 1997; 156: 536-541.

9. Gibson PG, Wlodarczyk J, Hensley MJ, et al. Epidemiological association of airway inflammation with asthma symptoms and airway hyperresponsiveness in childhood. Am J Respir Crit Care Med 1998; 158: 136-145.

10. Bhalla M, Turcios N, Aponte N, et al. Cystic fibrosis: scoring system with thin section CT. Radiology 1991; 179: 733-738. 
11. Greenberger PA. Allergic bronchopulmonary aspergillosis and fungoses. Clin Chest Med 1988; 9: 599-610.

12. Kauffman F, Tomee JFC, Van-der-Werf TS, de-Monchy JGR, Koeter GK. Review of fungus induced asthmatic reactions. Am J Respir Crit Care Med 1995; 151: 21092116.

13. Greenberger PA, Smith LJ, Hsu CC, Roberts M, Liotta JL. Analysis of bronchoalveolar lavage in allergic bronchopulmonary aspergillosis: divergent responses of antigen specific antibodies and total IgE. $J$ Allergy Clin Immunol 1988; 82: 164-170.

14. Kauffman HF, Koeter GH, van-der-Heide S, de-Monchy JG, Kloprogge E, de-Vries K. Cellular and humoral observations in a patient with allergic bronchopulmonary aspergillosis during a non-asthmatic exacerbation. $J$ Allergy Clin Immunol 1989; 83: 829-838.

15. Olivieri D, Pesci A, Bertorelli G. Eosinophilic alveolitis in immunologic interstial lung disorders. Lung 1990; 168 (Suppl.): 164-170.

16. Walker C, Bauer W, Braun RK, et al. Activated T cells and cytokines in bronchoalveolar lavages from patients with various lung diseases associated with eosinophilia. Am J Respir Crit Care Med 1994; 150: 1038-1048.

17. Bosken CH, Myers JL, Greenberger PA, Katzenstein AL.
Pathological features of allergic bronchopulmonary aspergillosis. Am J Surg Pathol 1988; 12: 216-222.

18. Cai Y, Carty K, Henry RL, Gibson PG. Persistence of sputum eosinophilia in children with controlled asthma when compared with healthy children. Eur Respir J 1998; 11: 848-853.

19. Cai Y, Carty K, Gibson PG, Henry RJ. Comparison of sputum processing techniques in cystic fibrosis. Pediatr Pulmonol 1996; 22: 402-407.

20. Gibson PG, Woolley KL, Carty K, Muree-Allen K, Saltos $\mathrm{N}$. Induced sputum eosinophil cationic protein measurement in asthma and chronic obstructive airway disease. Clin Exp Allergy 1998; 28: 1081-1088.

21. Malo JL, Longbottom J, Mitchell J, Hawkins R, Pepys J. Studies in chronic allergic bronchopulmonary aspergillosis. 3. Immunological findings. Thorax 1977; 32: 269274.

22. Kiely JL, Spense L, Henry M, Hurley MT, Kelleher N, Bredin CP. Chest radiographic staging in allergic bronchopulmonary aspergillosis: relationship with immunological findings. Eur Respir J 1998; 12: 453-458.

23. Wang JL, Patterson R, Roberts M, Ghory AC. The management of allergic bronchopulmonary aspergillosis. Am Rev Respir Dis 1979; 120: 87-92. 